\title{
TRPV1 activation improves exercise endurance and energy metabolism through PGC-1a upregulation in mice
}

\author{
Zhidan Luo ${ }^{1, *}$, Liqun Ma ${ }^{1, *}$, Zhigang Zhao ${ }^{1}$, Hongbo $\mathrm{He}^{1}$, Dachun Yang ${ }^{1}$, Xiaoli Feng ${ }^{1}$, Shuangtao Ma ${ }^{1}$, \\ Xiaoping Chen ${ }^{1}$, Tianqi Zhu ${ }^{1}$, Tingbing Cao ${ }^{1}$, Daoyan Liu ${ }^{1}$, Bernd Nilius ${ }^{2}$, Yu Huang ${ }^{3}$, Zhencheng Yan ${ }^{1}$, \\ Zhiming Zhu
}

${ }^{I}$ Center for Hypertension and Metabolic Diseases, Department of Hypertension and Endocrinology, Daping Hospital, Third Military Medical University, Chongqing Institute of Hypertension, Chongqing 400042, China; ${ }^{2} K U$ Leuven, Department of Molecular Cell Biology, Laboratory Ion Channel Research, Campus Gasthuisberg, Herestraat 49, B-3000 Leuven, Belgium; ${ }^{3}$ Institute of Vascular Medicine, Li Ka Shing Institute of Health Sciences and School of Biomedical Sciences, Chinese University of Hong Kong, Hong Kong, China

Impaired aerobic exercise capacity and skeletal muscle dysfunction are associated with cardiometabolic diseases. Acute administration of capsaicin enhances exercise endurance in rodents, but the long-term effect of dietary capsaicin is unknown. The capsaicin receptor, the transient receptor potential vanilloid 1 (TRPV1) cation channel has been detected in skeletal muscle, the role of which remains unclear. Here we report the function of TRPV1 in cultured C2C12 myocytes and the effect of TRPV1 activation by dietary capsaicin on energy metabolism and exercise endurance of skeletal muscles in mice. In vitro, capsaicin increased cytosolic free calcium and peroxisome proliferator-activated receptor- $\gamma$ coactivator-1 $\alpha$ (PGC-1 $\alpha$ ) expression in $\mathrm{C} 2 \mathrm{C} 12$ myotubes through activating TRPV1. In vivo, PGC$1 \alpha$ in skeletal muscle was upregulated by capsaicin-induced TRPV1 activation or genetic overexpression of TRPV1 in mice. TRPV1 activation increased the expression of genes involved in fatty acid oxidation and mitochondrial respiration, promoted mitochondrial biogenesis, increased oxidative fibers, enhanced exercise endurance and prevented high-fat diet-induced metabolic disorders. Importantly, these effects of capsaicin were absent in TRPV1-deficient mice. We conclude that TRPV1 activation by dietary capsaicin improves energy metabolism and exercise endurance by upregulating PGC-1 $\alpha$ in skeletal muscles. The present results indicate a novel therapeutic strategy for managing metabolic diseases and improving exercise endurance.

Keywords: TRPV1; exercise endurance; PGC-1 $\alpha$; skeletal muscle; energy metabolism

Cell Research (2012) 22:551-564. doi:10.1038/cr.2011.205; published online 20 December 2011

\section{Introduction}

Epidemiological studies suggest that exercise leads to lower blood pressure, reduced adiposity and improvements in dyslipidemia, insulin sensitivity and endothelial function [1]. Energy metabolism and exercise endurance, which directly influence cardiometabolic risk factors, are primarily driven by skeletal muscle, a major mass

\footnotetext{
*These two authors contributed equally to this work. Correspondence: Zhiming Zhu ${ }^{\mathrm{a}}$, Zhencheng Yan ${ }^{\mathrm{b}}$

${ }^{\text {a } T e l: ~+86-23-68767849 ; ~ F a x: ~+86-23-68705094 ~}$

E-mail: zhuzm@yahoo.com

${ }^{b}$ E-mail: zhenchengyan@sina.com

Received 30 August 2010; revised 7 July 2011; accepted 10 August 2011; published online 20 December 2011
}

peripheral tissue [2]. Impaired aerobic exercise capacity and skeletal muscle dysfunction appear to be associated with metabolic diseases, such as obesity and diabetes. Increasing oxidative fibers and exercise endurance can improve insulin action and prevent weight gain [3-5]. In this context, many attempts have been made to improve endurance capacity with nutritional regimens that promote fatty acid oxidation and muscle remodeling [6].

Capsaicin, the major pungent ingredient in hot pepper, is a highly selective agonist for transient receptor potential vanilloid 1 (TRPV1), a nonselective cation channel that primarily exists in sensory neurons and responds to noxious stimuli [7-8]. TRPV1 is also present in nonneuronal tissues, such as adipose tissue and skeletal muscle $[9,10]$. Our previous study showed that capsaicin inhib- 
ited adipogenesis through a TRPV1-mediated $\mathrm{Ca}^{2+}$ influx in adipocytes and chronic capsaicin administration prevented obesity in mice [11]. More recently, we showed that TRPV1 activation by dietary capsaicin improved endothelium-dependent vasorelaxation and regulated blood pressure in rats [12]. Acute oral administration of capsaicin enhanced fatty acid utilization and endurance capacity in rodents, and increased oxygen consumption in humans [13-15]. However, the long-term effects of dietary capsaicin have not been studied yet and whether capsaicin mediates these effects through skeletal muscle TRPV1 activation is still unclear.

Adult skeletal muscle shows plasticity and can convert into different fiber types in response to exercise training. The conversion of muscle fiber from glycolytic type II (fast fiber) to the more oxidative type I (slow fiber) is likely to be mediated by a $\mathrm{Ca}^{2+}$-signaling mechanism that involves calcineurin, calmodulin-dependent kinase and the transcriptional cofactor peroxisome proliferator-activated receptor- $\gamma$ coactivator-1 $\alpha$ (PGC-1 $\alpha)$ [16-18]. PGC-
A

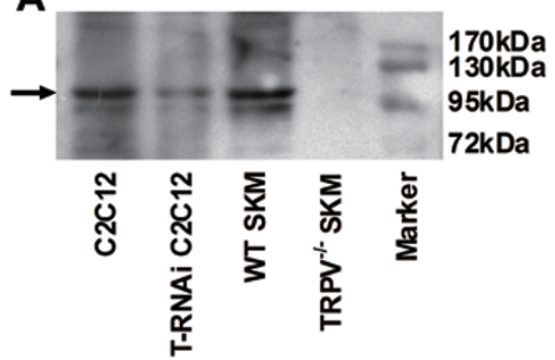

C

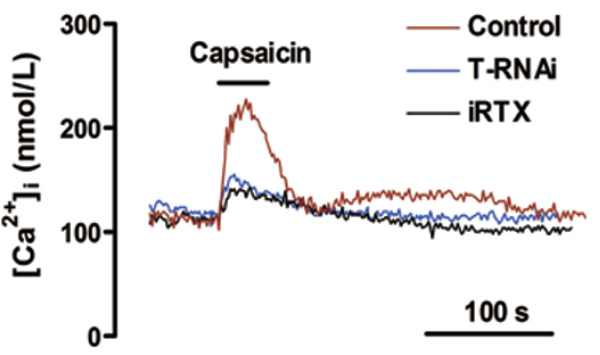

E

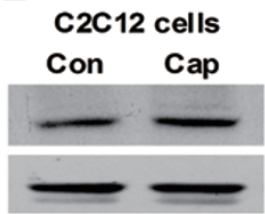

TRPV1

$\beta$-actin

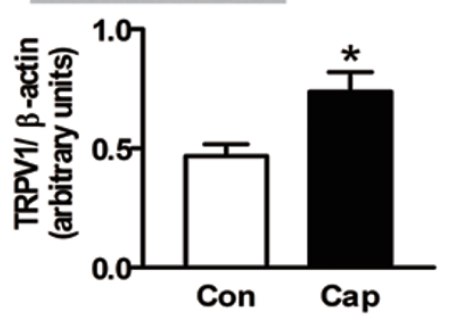

B

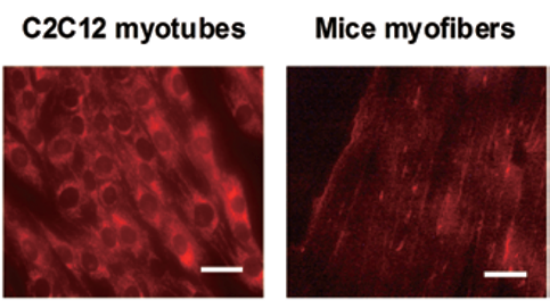

D



$\mathbf{F}$


Figure 1 TRPV1 characterization in skeletal muscles. (A) Immunoblot of TRPV1 in skeletal muscle (SKM) from wild-type (WT) and TRPV1 knockout (TRPV1 ${ }^{-1-}$ ) mice and in C2C12 myotubes with or without TRPV1 RNAi (T-RNAi). The arrowhead indicates the band corresponding to TRPV1 protein. (B) TRPV1 localization in C2C12 myotubes and mice myofibers was shown with immunofluorescence. Bar $=50 \mu \mathrm{m}$. (C, D) Representative curves (C) and summary data (D) showing capsaicin $(100 \mathrm{nM})$-induced $\left[\mathrm{Ca}^{2+}\right]_{i}$ changes in cells with or without T-RNAi and cells pretreated with the specific TRPV1 inhibitor iRTX (1 $\mu \mathrm{M}$ ) for 5 min. ${ }^{* *} P<0.01$ vs control. (E) Immunoblot of TRPV1 in C2C12 myotubes with (Cap) or without (Con) capsaicin (100 nM) treatment for 24 h. ${ }^{*} P<0.05$ vs Con. (F) Immunoblot of TRPV1 in skeletal muscles of WT mice with (Cap) or without (Con) 4 months of capsaicin administration. ${ }^{*} P<0.05$ vs Con. Summary data are means \pm S.E.M. for three to four independent experiments. 
$1 \alpha$ is a principal regulator of the expression of genes involved in mitochondrial biogenesis, respiratory function, and carbohydrate and lipid metabolism [16-18]. It also participates in the pathogenesis of obesity, diabetes and cardiomyopathy $[16,19]$. Thus, we hypothesize that TRPV1 activation by capsaicin might improve energy metabolism and endurance capacity in skeletal muscles through $\mathrm{Ca}^{2+}$-dependent PGC-1 $\alpha$ upregulation.

\section{Results}

\section{TRPV1 characterization in skeletal muscles}

We examined TRPV1 protein expression in skeletal muscles from WT and TRPV $1^{-/}$mice and in cultured C2C12 myotubes (Figure 1A). TRPV1 protein was abundant in skeletal muscles of WT mice but was absent in $\mathrm{TRPV}^{-/-}$mice. TRPV1 RNA interference (RNAi) reduced the expression of TRPV1 in C2C12 cells by $>75 \%$ (Figure 1A). Immunofluorescence staining showed that TRPV1 was located in both the cytoplasm and plasma membranes of myocytes (Figure 1B). To determine the function of TRPV1 channels in myotubes, we examined the intracellular free calcium concentration $\left(\left[\mathrm{Ca}^{2+}\right]_{\mathrm{i}}\right)$. Acute capsaicin stimulation caused a marked increase of $\left[\mathrm{Ca}^{2+}\right]_{i}$ in control myotubes (Figure 1C). A specific blockade of TRPV1 by 5'-iodo-resiniferatoxin (iRTX) and TRPV1 RNAi inhibited the capsaicin-induced $\left[\mathrm{Ca}^{2+}\right]$ i increase by $75 \%$ and $72 \%$, respectively (Figure $1 \mathrm{C}$ and 1D). TRPV1 was upregulated by capsaicin treatment. In vitro, TRPV1 protein expression was higher in $\mathrm{C} 2 \mathrm{C} 12$ cells treated with capsaicin for $24 \mathrm{~h}$ compared to cells without capsaicin treatment (Figure 1E). In vivo, administering a capsaicin diet to mice for 4 months increased the TRPV1 expression in skeletal muscles (Figure 1F). These results indicate that TRPV1 is expressed in cultured myotubes and mouse skeletal muscles, which can be activated and upregulated by capsaicin treatment.

TRPV1 activation increases $P G C-1 \alpha$ expression and mitochondrial biogenesis in a $\mathrm{Ca}^{2+}$-dependent manner

What is the physiological significance of TRPV1 channels in myotubes? $\mathrm{Ca}^{2+}$ and several $\mathrm{Ca}^{2+}$-dependent signaling pathways are involved in the induction of PGC$1 \alpha$ and its target genes [18, 20, 21]. PGC- $1 \alpha$ is a master regulator of lipid and glucose metabolism, mitochondrial biogenesis and muscle remodeling [22]. Thus, we examined whether capsaicin exposure could upregulate PGC- $1 \alpha$ through TRPV1-mediated $\mathrm{Ca}^{2+}$ increases. The PGC-1 $\alpha$ expression was elevated after 24 -h capsaicin treatment while this increase was reversed by the presence of iRTX and the intracellular $\mathrm{Ca}^{2+}$ chelator BAPTA (1,2-bis(o-aminophenoxy)ethane-N,N,N',N'-tetraacetic acid) (Figure 2A). It is well documented that CD36 and the muscle isoform of carnitine palmityl transferase 1 are important fatty acid transporter and key enzyme in mitochondrial fatty acid oxidation [23-27]. Here we showed that TRPV1 activation by capsaicin significantly upregulated CD36 and carnitine palmityl transferase 1, which was also abolished by iRTX (Figure 2B). In contrast to fatty acid oxidation, glycolysis is reported to be inhibited by PGC-1 $\alpha$ activation [28]. We showed here that hexokinase type II enzyme, a critical enzyme in glycolytic process [29], was significantly decreased by capsaicin and restored by the presence of iRTX (Figure 2B). To evaluate the effects of capsaicin on mitochondrial biogenesis and respiration, we detected cytochrome $\mathrm{C} 1$ and surfeit 1, the subunits of complex III and IV [30] in myotubes. Capsaicin increased the expressions of these mitochondrial genes, which were inhibited by iRTX (Figure 2C). We further assessed the mitochondrial mass by staining cells with a mitochondrion-selective dye and showed that the mitochondrial content was increased in capsaicin-treated cells compared with the control (Figure 2D). Likewise, the ATP production in myotubes was elevated by capsaicin treatment (Figure 2E). The effects of capsaicin on mitochondrial biogenesis and ATP production were both markedly attenuated by iRTX and BAPTA (Figure 2D and 2E). These in vitro results suggest that TRPV1 activation by capsaicin increases the PGC- $1 \alpha$ expression, mitochondrial biogenesis and ATP production in myotubes in a $\mathrm{Ca}^{2+}$-dependent manner.

Activation of TRPV1 by dietary capsaicin upregulates $P G C-1 \alpha$ and improves mitochondrial function and muscle remodeling

$\mathrm{WT}$ and $\mathrm{TRPV}^{-/-}$mice were given either a regular chow diet or a diet containing capsaicin for 4 months. In agreement with the in vitro experiments, administration of capsaicin significantly increased the expression of PGC-1 $\alpha$, CD36 and carnitine palmityl transferase 1, but reduced the hexokinase type II enzyme expression in skeletal muscles (Figure 3A and 3B). The mitochondrial respiratory complex III and IV subunits and troponin I, the characteristic protein for oxidative slow fibers, were increased in mice on capsaicin diet compared to control mice (Figure 3A, 3B and 3D). By contrast, the effects of capsaicin consumption on mitochondrial respiratory complexes and troponin were absent in $\mathrm{TRPV}^{-/-}$mice (Figure 3A-3D). The mitochondrial content in skeletal muscles was increased by dietary capsaicin in WT mice, but not in TRPV $1^{--}$mice (Figure 3E and Supplementary information, Figure S1). The respiratory control index was markedly increased resulting from enhanced state-3 respiration and unchanged state- 4 respiration in isolated 
mitochondria from WT mice on capsaicin diet, which was not observed in TRPV1 $1^{-/}$mice (Figure 3F). Skeletal muscle fiber types are partly determined by mitochondrial content, oxidative respiratory capacity and the balance between lipid oxidation and glycolysis [3, 31]. We then identified the fiber types in gastrocnemius muscles and showed that there were more Type I (oxidative) fibers and fewer Type II (glycolytic) fibers in capsaicin-treated mice
A Con Cap Capt Cap+

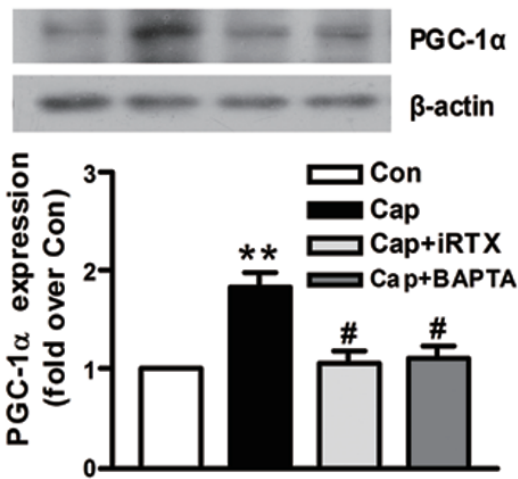

C Con Cap Cap+iRTX

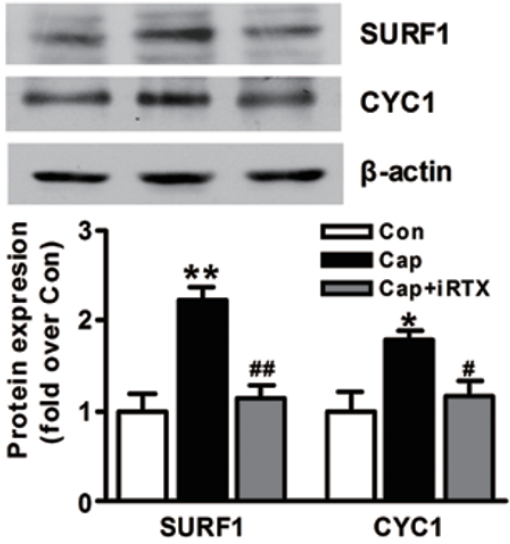

E

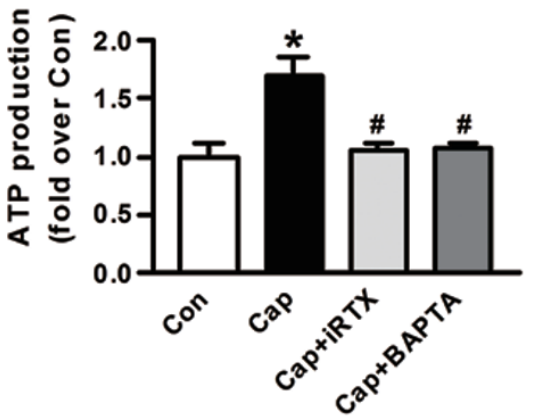

B Con Cap Cap+iRTX
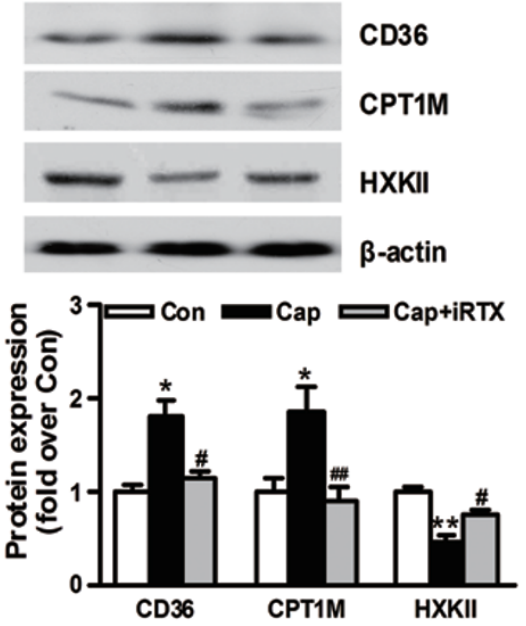

D Con Cap

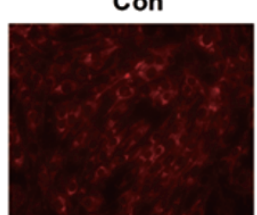

Cap+iRTX

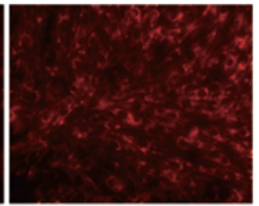

Cap+BAPTA
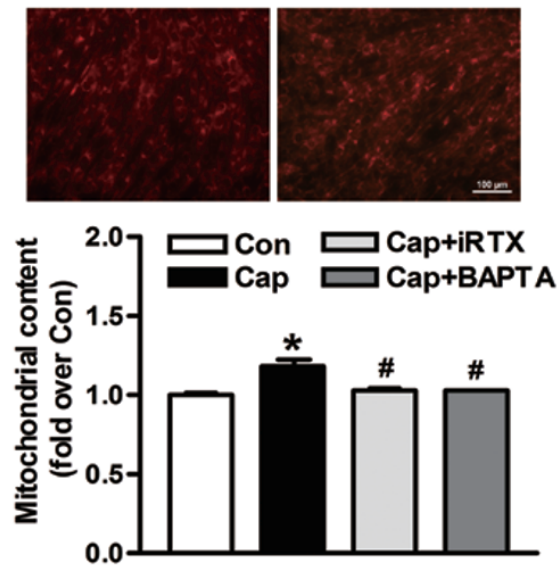

Figure 2 TRPV1 activation increases PGC-1 $\alpha$ expression and mitochondrial biogenesis in a $\mathrm{Ca}^{2+}$-dependent manner. (A) Immunoblot of PGC-1 $\alpha$ in $\mathrm{C} 2 \mathrm{C} 12$ myotubes treated with capsaicin $(100 \mathrm{nM})$ in the presence or absence of the TRPV1 inhibitor $\operatorname{iRTX}(1 \mu \mathrm{M})$ or the intracellular $\mathrm{Ca}^{2+}$ chelator BAPTA $(10 \mu \mathrm{M})$. (B, C) Protein expression of genes involved in fatty acid oxidation, glycolysis (B) and mitochondrial respiration (C) in myotubes treated with capsaicin (100 nM) in the presence or absence of $\mathrm{iRTX}(1 \mu \mathrm{M})$. (D, E) Mitochondrial content (D) and ATP production (E) in myotubes. C2C12 cells were treated with capsaicin $(100 \mathrm{nM})$ in the presence or absence of $\mathrm{iRTX}(1 \mu \mathrm{M})$ or BAPTA $(10 \mu \mathrm{M})$ for $24 \mathrm{~h}$. Data are means \pm S.E.M. for three independent experiments. Con, control; Cap, capsaicin. ${ }^{*} P<0.05$, ${ }^{* *} P<0.01$ vs Con. ${ }^{\#} P<0.05,{ }^{\#} P<0.01$ vs Cap. 

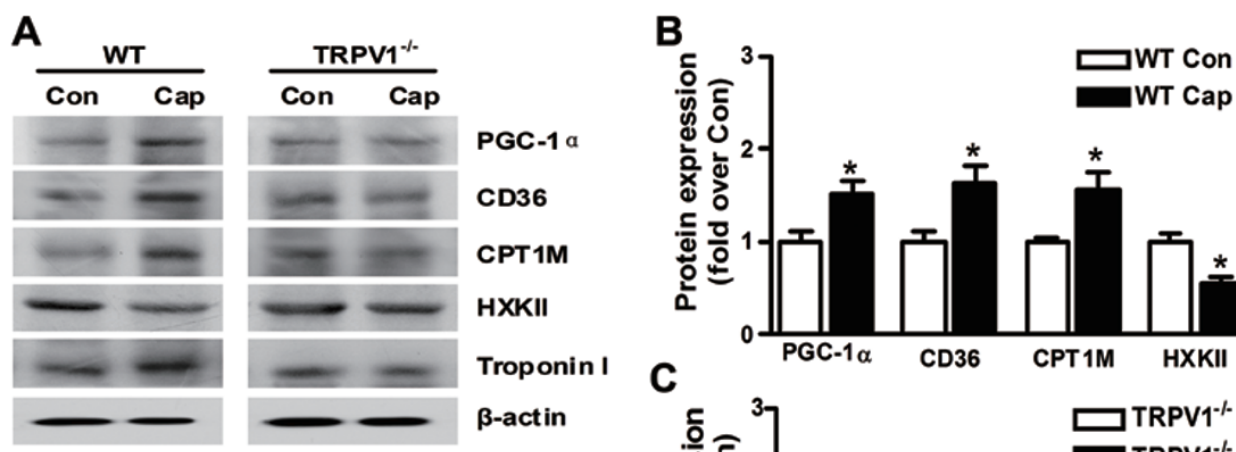

D

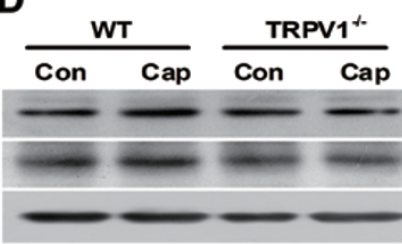

SURF1

CYC1

B-actin
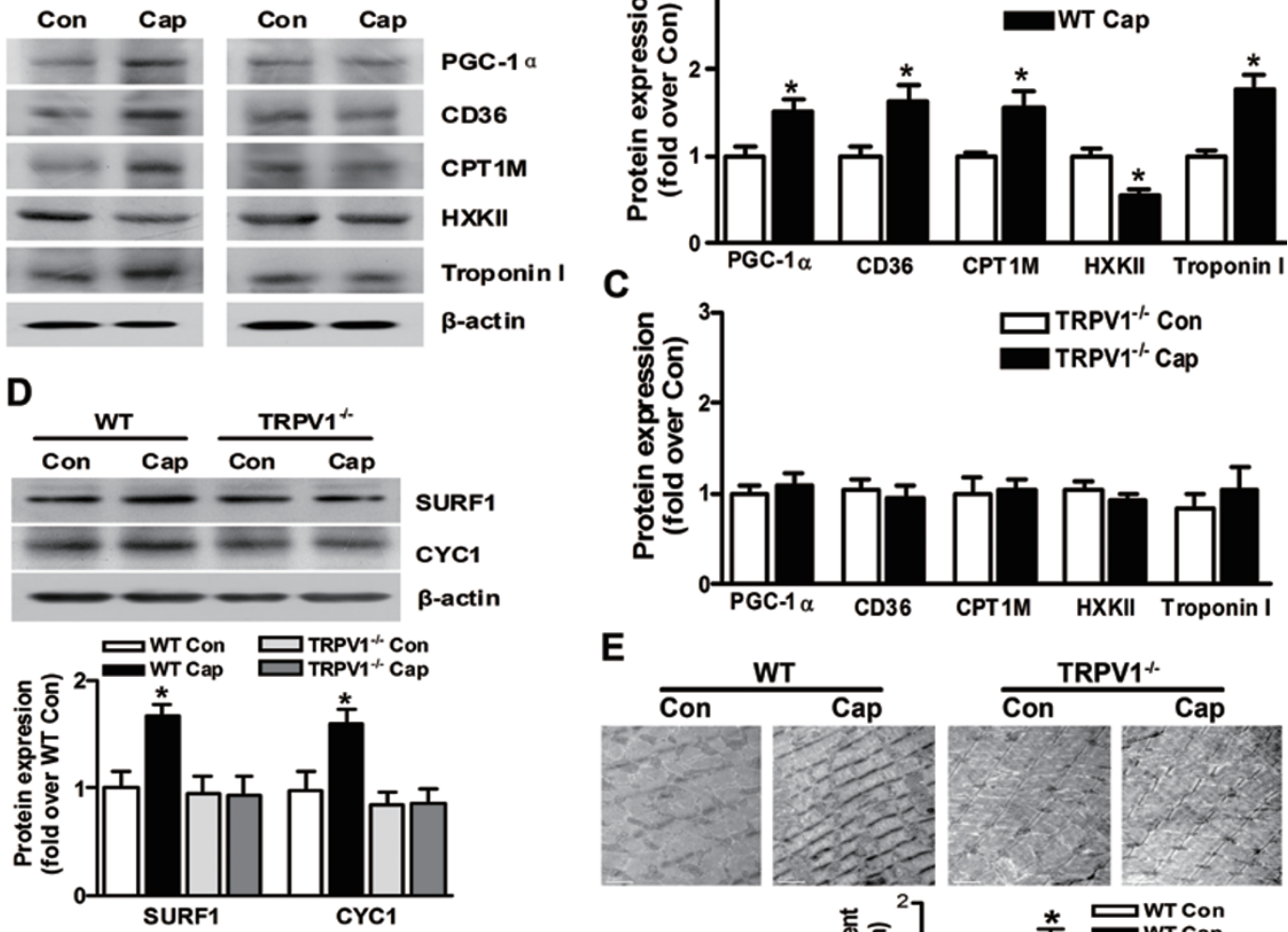

$\mathbf{E}$
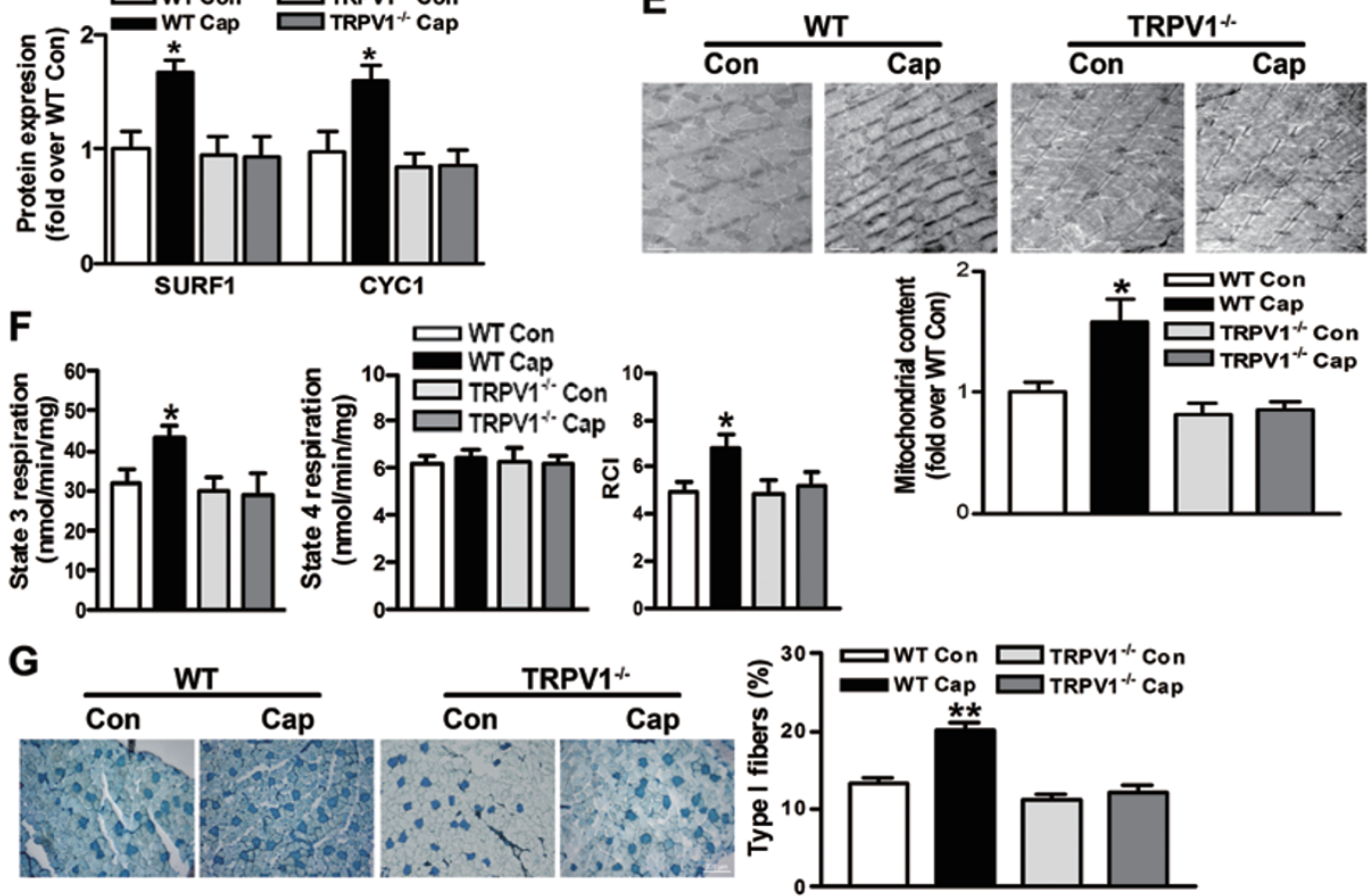

Figure 3 Activation of TRPV1 by dietary capsaicin up-regulates PGC-1 $\alpha$ and improves mitochondrial function and muscle remodeling. (A-D) Protein expression of genes involved in fatty acid oxidation, glycolysis, fiber specification (A-C) and mitochondrial respiration (D) in skeletal muscles from WT and TRPV1 $1^{-1-}$ mice fed a regular diet (Con) or a capsaicin diet (Cap). (E) Mitochondrial mass in gastrocnemius muscle shown by transmission electron microscopy. (F) State 3 and state 4 respiration and respiratory control index $(\mathrm{RCl})$ in mitochondria isolated from fresh quadriceps femoris muscles. (G) Percentage of type I fibers in gastrocnemius muscle shown by the metachromatic staining. Oxidative (Type I) fibers were stained dark blue. Data are means \pm S.E.M. $n=3-8 .{ }^{*} P<0.05,{ }^{* *} P<0.01$ vs WT.

compared to control mice (Figure 3G). There was no difference in fiber type percentages between TRPV ${ }^{-/-}$mice with and without dietary capsaicin consumption (Figure $3 \mathrm{G})$. These results indicate that chronic activation of
TRPV1 with dietary capsaicin upregulates PGC-1 $\alpha$, promotes mitochondrial biogenesis and oxidative respiratory capacity, and consequently improves skeletal muscle fiber transformation. 

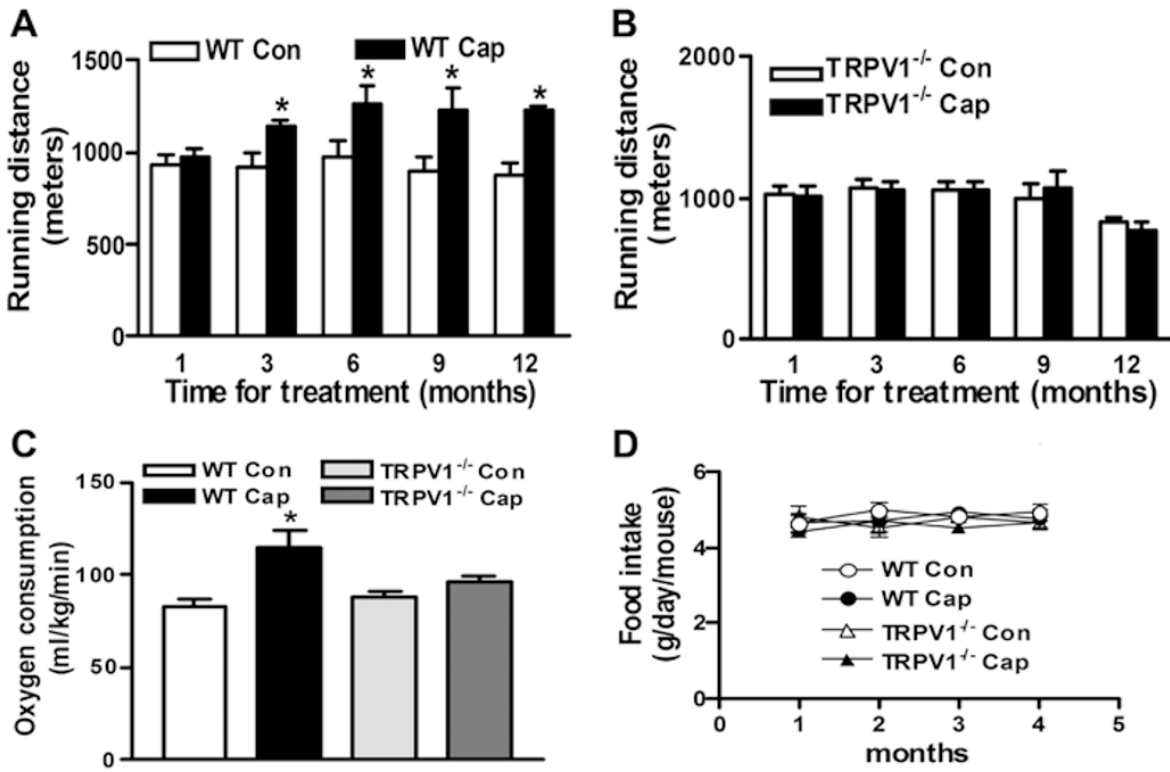

D
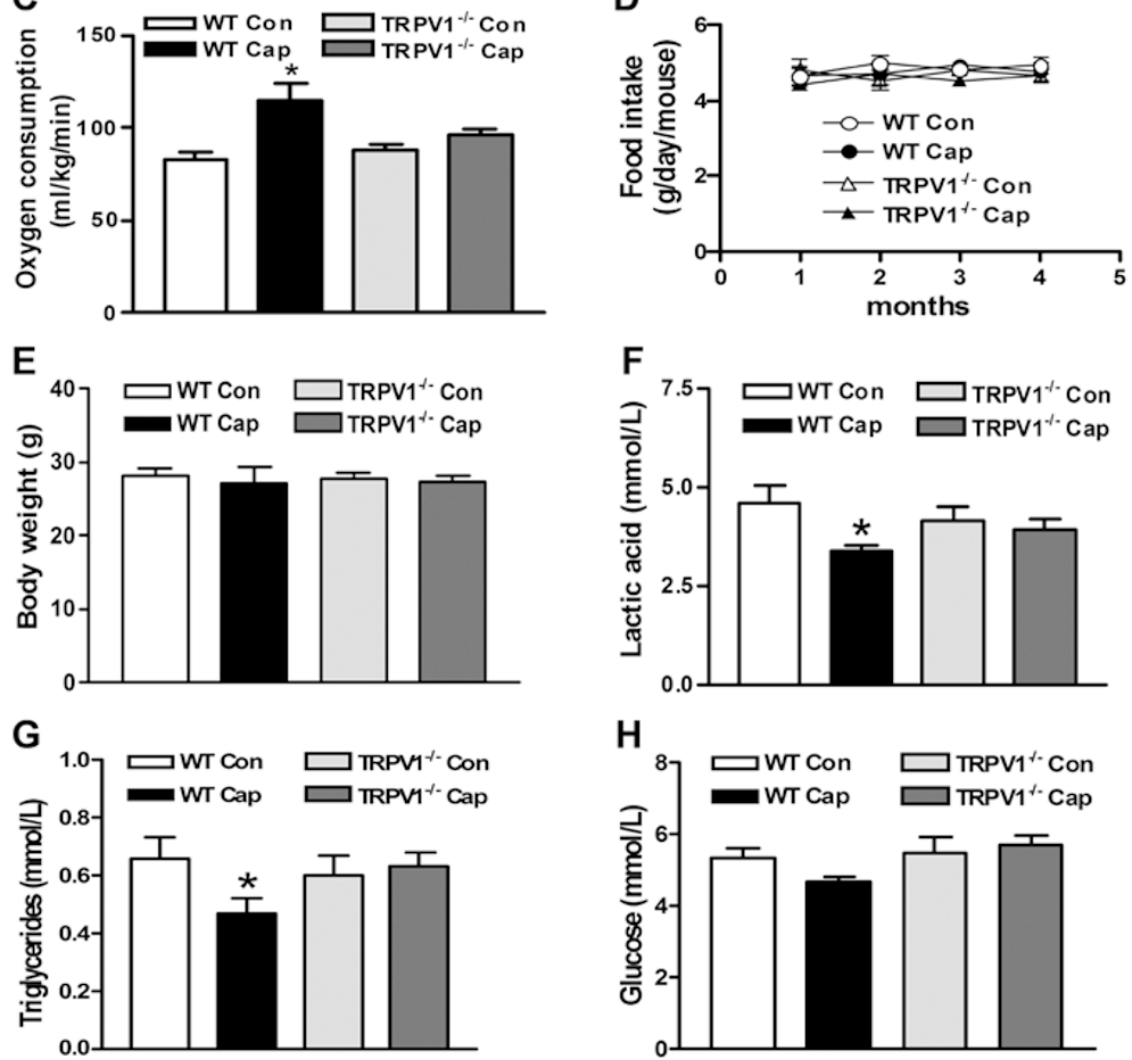

Figure 4 Dietary capsaicin enhances exercise endurance and reduces blood lactic acid and triglycerides through TRPV1 activation. (A, B) Exercise endurance test with treadmill exercise. WT (A) and TRPV1 $1^{-/-}$mice (B) on a regular diet (Con) and a capsaicin diet (Cap) for indicated months were tested. Running distance before reaching exhaustion was recorded. Data are presented as the means \pm S.E.M. for 6-10 mice. ${ }^{*} P<0.05$ vs Con. (C) Oxygen consumption (ml $\left./ \mathrm{kg} / \mathrm{min}\right)$ examined when mice ran for $30 \mathrm{~min}$ at a speed of $10 \mathrm{~m} / \mathrm{min}$. (D) Average daily food intake (g/d) per mouse determined during the last week of each month. (E-H) Body weight and blood levels of lactic acid, triglycerides and glucose. Mice were treated with control or capsaicin diet for 4 months. Data are means \pm S.E.M. for 5-9 mice. ${ }^{*} P<0.05$ vs Con.

Dietary capsaicin enhances exercise endurance and reduces blood lactic acid and triglycerides through TRPVI activation

We further examined whether dietary capsaicin could improve exercise endurance and the related biochemical parameters through TRPV1 activation. Running endurance was performed with treadmill tests and oxygen consumption was measured in vivo. After 3-month cap- saicin treatment, mice ran about 300 meters more before reaching exhaustion than the control (Figure 4A). Similar results were obtained for WT mice treated with capsaicin for 6, 9 and 12 months (Figure 4A). The oxygen consumption was also increased by capsaicin treatment in WT mice (Figure 4C). By contrast, administration of capsaicin did not affect exercise endurance and oxygen consumption in TRPV1 ${ }^{-/}$mice (Figure $4 \mathrm{~B}$ and $4 \mathrm{C}$ ). The 
daily food intake per month indicated that all mice tolerated the dietary capsaicin well (Figure 4D). The body weights were similar among all the groups after 4-month treatment (Figure 4E). Blood lactic acid, a product of glycolysis, is one of the key determinants for endurance performance [31]. Capsaicin treatment significantly lowered the blood lactic acid and triglyceride levels in WT mice, but not in TRPV1 $1^{-/}$mice (Figure $4 \mathrm{~F}$ and $4 \mathrm{G}$ ). The blood glucose also showed a reducing tendency in capsaicin-treated mice (Figure 4H). These results support the notion that activation of TRPV1 by chronic dietary capsaicin enhances exercise endurance and improves systemic metabolic status.

Transgenic TRPV1 gene increases PGC-1 $\alpha$ expression, oxidative fibers and exercise endurance

We then employed TRPV1-transgenic mice (TRPV1$\operatorname{tg})$ to further investigate whether TRPV1 mediated the
A

$$
\text { WT TRPV1-tg }
$$


E

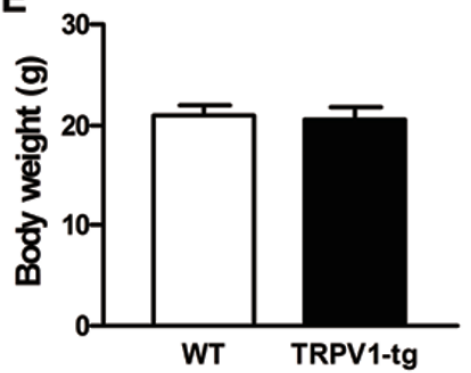

B


TRPV1-tg
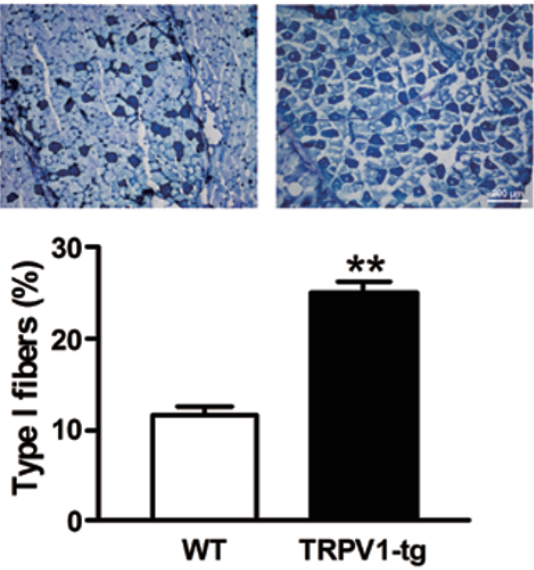

D

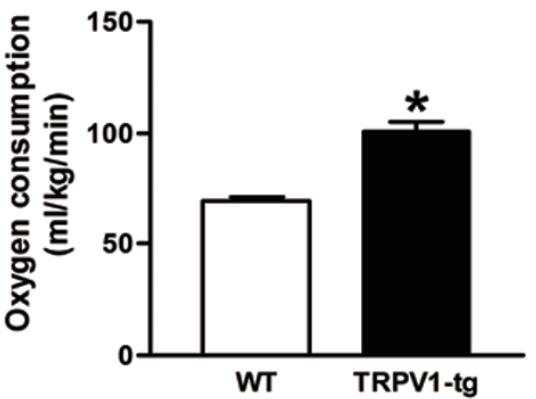

$\mathbf{F}$

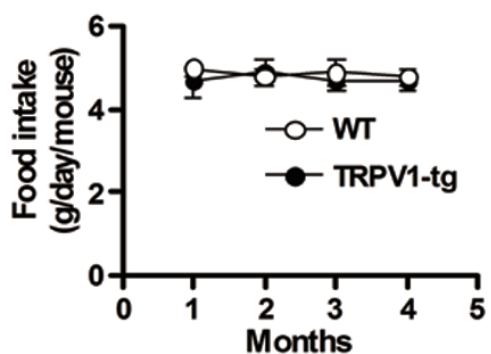

Figure 5 Transgenic TRPV1 gene increases PGC-1 $\alpha$ expression, oxidative fibers and exercise endurance. (A) Immunoblot of TRPV1 and PGC-1 $\alpha$ in skeletal muscle from TRPV1-tg and their wild-type littermates (WT). ${ }^{*} P<0.05,{ }^{* *} P<0.01$ vs WT. (B) Metachromatic staining of gastrocnemius muscle. Type I fibers were stained dark blue. (C) Exercise endurance, (D) oxygen consumption, (E) body weight and (F) average daily food intake in TRPV1 transgenic mice and their WT littermates. ${ }^{*} P<0.05$, ${ }^{* *} P<0.01$ vs WT. Mice were fed a regular diet until they reached 6 months of age. Data are means \pm S.E.M. for 3 mice. 
increase of PGC- $1 \alpha$ expression, oxidative fibers and exercise endurance. Compared with WT littermates, TRPV1-tg mice had significantly higher expression of TRPV1 and PGC-1 $\alpha$ in skeletal muscles (Figure 5A). The mitochondrial content (Supplementary information, Figure S2) and the percentage of oxidative type I fibers (Figure 5B) in skeletal muscles were markedly increased in TRPV1-tg mice compared with WT littermates. Importantly, TRPV1-tg mice showed an impressive exercise performance. TRPV1-tg mice ran almost twice the distance of their WT littermates (Figure 5C). Likewise, the oxygen consumption of TRPV1-tg mice was higher than that of WT littermates (Figure 5D). However, the body weight and daily food intake did not differ between
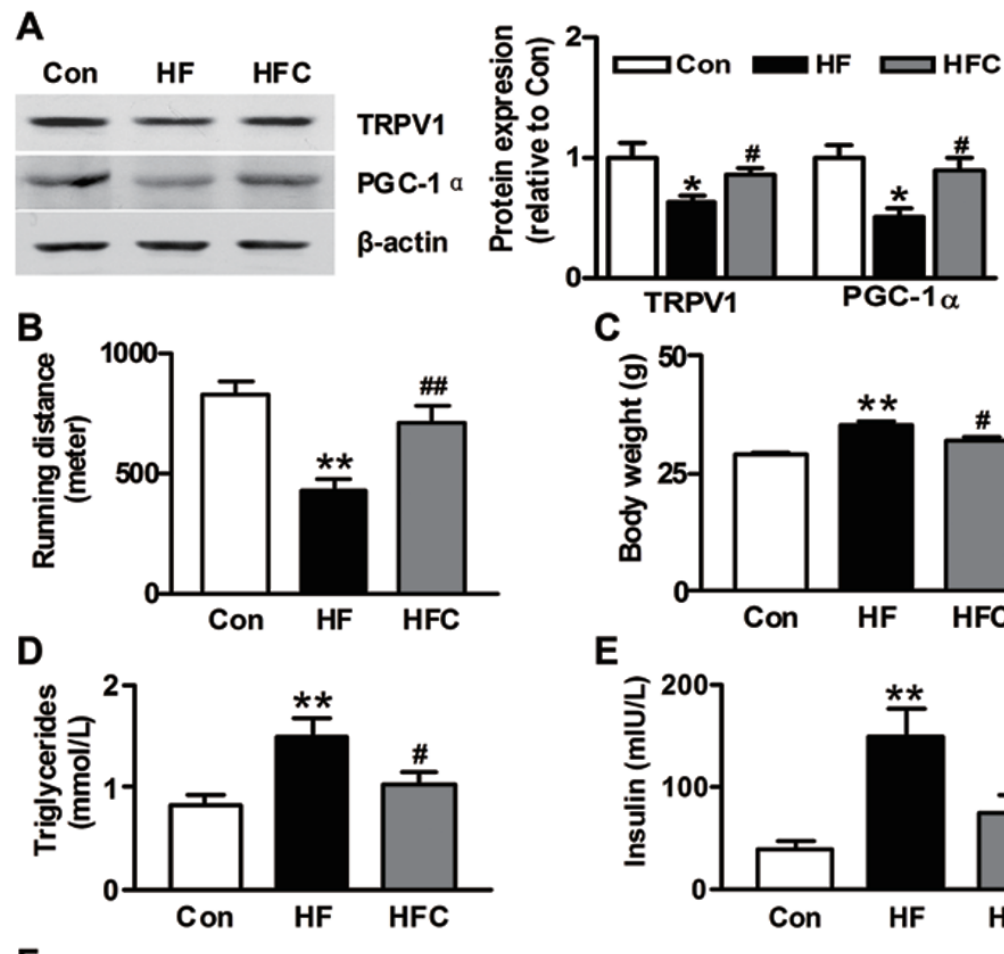

C
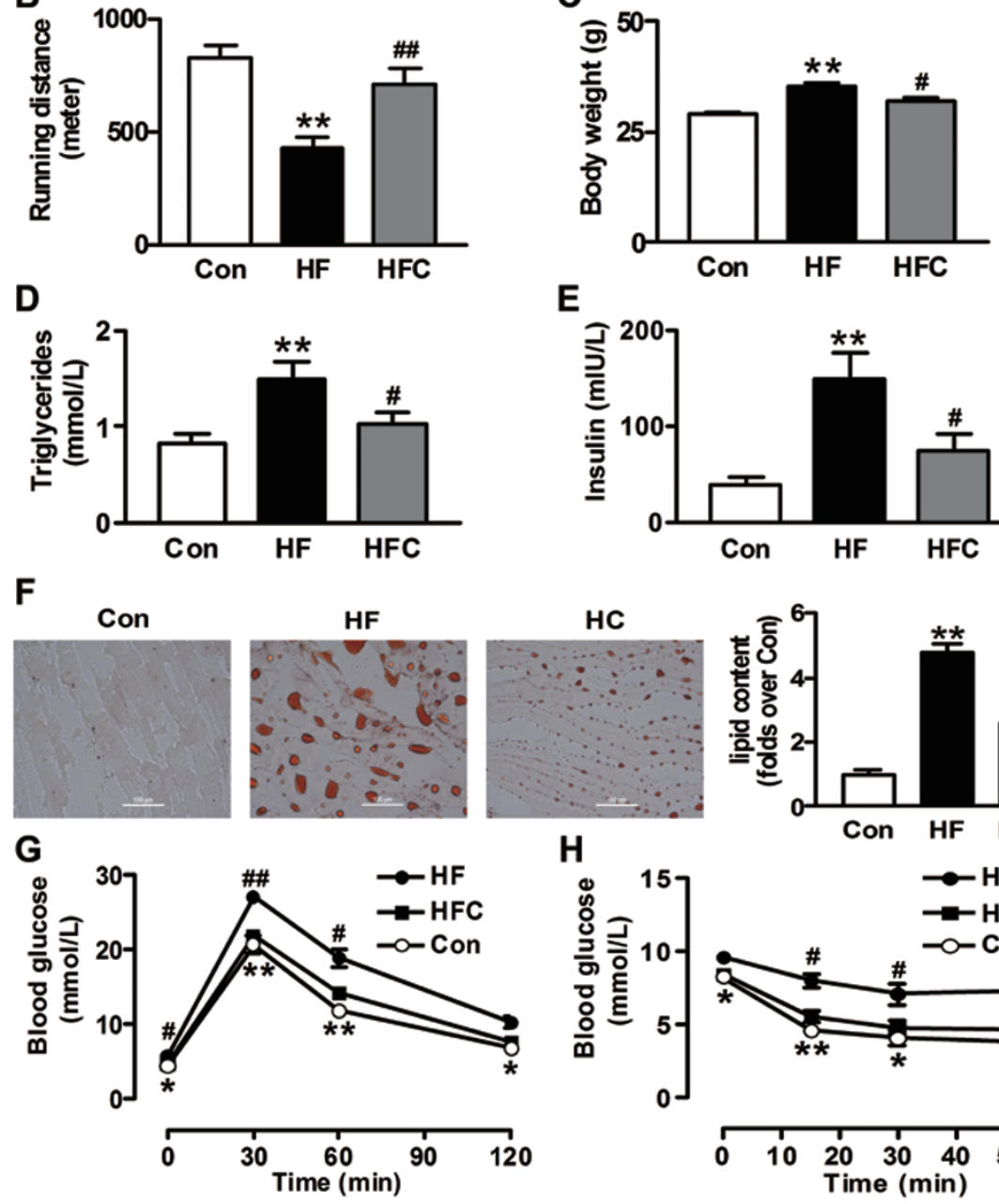

E

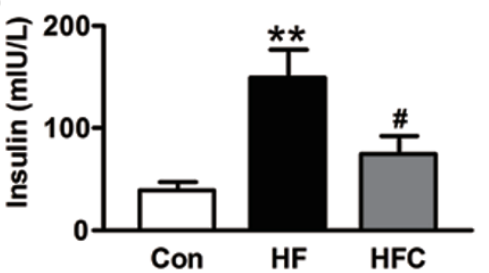

HC


Figure 6 TRPV1 activation restores the HFD-induced PGC-1 $\alpha$ downregulation, endurance impairment and metabolic disorders. (A) Immunoblot of TRPV1 and PGC-1a in skeletal muscle from WT mice on normal diet (Con) and HFD without (HF) or with capsaicin supplementation (HFC). (B-E) Running endurance (B), body weight (C) and blood levels of triglycerides (D) and insulin (E) in mice. (F) Lipid contents of quadriceps femoris muscles shown by Oil-red $\mathrm{O}$ staining. ${ }^{*} P<0.05,{ }^{* *} P<0.01$ vs Con; ${ }^{\#} P<0.05,{ }^{\#} P<0.01$ vs HF. (G, H) Intraperitoneal glucose tolerance test (IPGTT) and intraperitoneal insulin tolerance test (IPITT). ${ }^{\star} P<0.05,{ }^{* *} P<0.01$ vs HF; ${ }^{\#} P<0.05,{ }^{\# \#} P<0.01$ vs HFC. Data are means \pm S.E.M. for three to six mice. 
the two groups (Figure 5E and 5F). These results suggest that genetic TRPV1 overexpression produces similar metabolic changes as in capsaicin-treated mice.

TRPV1 activation restores the high-fat diet (HFD)induced $P G C-1 \alpha$ downregulation, endurance impairment and metabolic disturbance

To clarify whether dietary capsaicin prevents metabolic disorders induced by HFD, mice on HFD were treated with capsaicin for 4 months. Compared with control mice, mice on HFD had lower expression of TRPV1 and PGC-1 $\alpha$ (Figure 6A), mitochondrial content (Supplementary information, Figure S3) and percentage of type I fibers (Supplementary information, Figure S4) in skeletal muscles, which were significantly restored by dietary capsaicin. As a result, mice on HFD had a severely impaired endurance capacity, which was largely prevented by dietary capsaicin (Figure 6B). In addition, capsaicin treatment ameliorated the HFD-induced obesity, hyperlipidemia and hyperinsulinemia (Figure 6C-6E). It was noted that the skeletal muscles of mice on HFD contain much more lipids than those of control mice, but the lipid content in skeletal muscles was markedly reduced in mice on HFD plus capsaicin (Figure 6F). The intraperitoneal glucose and insulin tolerance tests showed that capsaicin treatment significantly attenuated HFD-induced glucose intolerance and insulin resistance (Figure 6G and $6 \mathrm{H})$. The present results suggest that HFD-induced endurance impairment and metabolic disorders can be prevented by dietary capsaicin consumption through TRPV1-mediated PGC-1 $\alpha$ upregulation.

\section{Discussion}

Overwhelming evidence suggests that cardiometabolic diseases are closely associated with a lack of exercise and unhealthy eating habits. Recent studies show that aerobic exercise capacity inversely correlates with cardiovascular disease and all causes of mortality across populations [32-34]. Therefore, exercise and dietary interventions are highly recommended for the prevention of cardiometabolic diseases. Major findings of the present study show that dietary capsaicin consumption improves exercise endurance and energy metabolism through TRPV1-mediated PGC-1 $\alpha$ upregulation in skeletal muscles.

Most previous studies focused on TRPV1 channel properties and their neuronal effects. However, TRPV1 has recently been reported to play different roles in many types of cells and tissues. Besides the involvement of TRPV1 in adipogenesis and vascular relaxation [11-12], TRPV1 in neurons and smooth muscle plays opposite functional roles in the tissue-specific regulation of microvascular diameter [35]. The present study reveals for the first time that chronic TRPV1 activation induces PGC$1 \alpha$ expression and improves mitochondrial function in a $\mathrm{Ca}^{2+}$-dependent manner in the skeletal muscle. TRPV1 is expressed in both the sarcoplasmic reticulum and plasma membrane of mouse myotubes, which is in agreement with a previous report on rat skeletal muscle [10]. $\mathrm{Ca}^{2+}$ permeability is a basic property of TRPV1 channels. Capsaicin, a highly lipophilic TRPV1 agonist, is reported to exert its effects through TRPV1-mediated $\mathrm{Ca}^{2+}$ mobilization [9]. The present study shows that capsaicin induced a significant increase of $\left[\mathrm{Ca}^{2+}\right]_{\mathrm{i}}$ in myotubes, which was blocked by the specific TRPV1 antagonist iRTX. $\mathrm{Ca}^{2+}$ - and calmodulin-dependent protein kinase can activate the PGC-1 $\alpha$ gene promoter and induce PGC- $1 \alpha$ expression, thus contributing to mitochondrial biogenesis in myocytes $[18,21,36]$. We show that capsaicin exposure resulted in a significant increase in PGC- $1 \alpha$ expression, mitochondrial biogenesis and ATP production in cultured myotubes, which can be abolished by inhibition of TRPV1 or depletion of intracellular $\mathrm{Ca}^{2+}$. It is likely that the TRPV1-mediated $\mathrm{Ca}^{2+}$ increase is critical for the capsaicin-induced PGC-1 $\alpha$ expression in myotubes.

Several earlier studies showed that a single dose of capsaicin temporarily promoted oxygen consumption and lipid utilization and enhanced swimming capability in rodents, which was associated with sympathetic activation [13-14]. However, it is unclear whether chronic capsaicin consumption could enhance exercise endurance on a more permanent basis, whether TRPV1 in skeletal muscle is involved in these long-term changes and what the mechanisms might be. The present study shows that chronic dietary capsaicin continuously improved exercise endurance with no effect on food intake. We provide some mechanisms through which capsaicin treatment improves exercise endurance. First, the in vitro studies on cultured myotubes showed that capsaicin induced PGC- $1 \alpha$ expression, mitochondrial biogenesis and ATP production via TRPV1-mediated $\mathrm{Ca}^{2+}$ signals. Second, dietary capsaicin administration in mice increased the expression of TRPV1 and PGC- $1 \alpha$ in skeletal muscles, and consequently upregulated PGC- $1 \alpha$ target genes involved in fatty acid oxidation and mitochondrial respiration, and downregulated the genes involved in glycolysis. Third, dietary capsaicin increased the mitochondrial mass and respiratory function, and promoted a fiber transformation from glycolytic type II fibers to more oxidative type I fibers. It is known that type I fibers contain more mitochondria and produce more ATP from lipid oxidation but less from glycolysis compared to type II fibers, thus providing stable energy for a longer time with less lactic 
acid accumulation [3, 31, 37].

Muscle fiber specification and mitochondrial function have also been associated with metabolic diseases in addition to exercise performance. Obese and diabetic patients have reduced oxidative capacity, increased glycolytic capacity and a decreased percentage of type I fibers in muscles [38-39]. Increasing the percentage of oxidative fibers can lead to improved insulin action and reduced adipocyte size [3-5]. We showed that HFD not only led to various metabolic disorders but also led to downregulation of PGC- $1 \alpha$ and reduced percentage of type 1 fibers, which is consistent with previous studies by others [30]. PGC-1 $\alpha$ downregulation was supposed to contribute to both the progression of muscle dysfunction and metabolic disorders. Increased expression of PGC- $1 \alpha$ results in increased mitochondrial function and oxidative muscle fibers and protects from metabolic disease during aging [40, 41]. The present study shows that with improved endurance capacity, dietary capsaicin also ameliorated the HFD-induced obesity, insulin resistance and glucose and lipid disorders. Both of the benefits of capsaicin on exercise endurance and energy metabolism might be attributed to the restoration of mitochondrial function and lipid oxidative capacity via TRPV1-mediated PGC-1 $\alpha$ upregulation in skeletal muscles.

In addition, we showed that the TRPV1 is upregulated by capsaicin treatment in vitro and in vivo. We also found that exercise (Supplementary information, Figure S5) and HFD affect the protein expression of TRPV1 in muscles. It is known that TRPV1 can be activated by heat, protons and several endogenous lipid molecules, such as anandamide and eicosanoids [42]. Anandamide is the most abundant endogenous endocannabinoid and regulates systemic energy metabolism [43]. Anandamide in skeletal muscles participates in the regulation of TRPV1 function $[44,45]$. We showed that anandamide treatment significantly increased TRPV1 expression (Supplementary information, Figure S6). Thus, it is possible that some endogenous lipid signals could trigger TRPV1 in the context of muscle biology. However, this needs further investigation.

In conclusion, TRPV1 activation by dietary capsaicin improves energy metabolism and exercise endurance, which are likely driven by TRPV1-mediated $\mathrm{Ca}^{2+}$-dependent upregulation of PGC- $1 \alpha$ and its target genes involved in the mitochondrial respiration and fatty acid oxidation. The present study reveals a new role of TRPV1 in skeletal muscle function and energy metabolism. Our novel findings also suggest that dietary capsaicin supplementation can be a promising lifestyle modification strategy to improve skeletal muscle function and prevent cardiometabolic diseases.

\section{Materials and Methods}

\section{Cell culture}

The mouse skeletal muscle cell line C2C12 (ATCC, Manassas, VA, USA) was propagated in Dulbecco's modified Eagle's medium-high glucose supplemented with $10 \%$ fetal bovine serum. $\mathrm{C} 2 \mathrm{C} 12$ myoblasts were cultured for $48 \mathrm{~h}$ to reach confluence, and then the growth medium was changed to differentiation medium (Dulbecco's modified Eagle's medium supplemented with 2\% horse serum) to induce myogenic differentiation. Experiments were performed after 2 days in the differentiating medium. Dulbecco's modified Eagle's medium, fetal bovine serum and horse serum were purchased from Gibco Co. (Grand Island, NY, USA). Chemicals including capsaicin, iRTX and BAPTA were purchased from Sigma-Aldrich Co. (St Louis, MO, USA).

\section{TRPV1 gene silencing}

TRPV1 was selectively silenced with RNAi using a lentiviral system according to a previously published technique [11]. DNA templates for synthesizing silencing RNA were cloned into an expression plasmid for subsequent transfection. The coding sequence for TRPV1-targeting mRNA was selected using the siRNA Target Finder and Design Tool from Ambion. The potential target sequence was subjected to a BLAST search against mouse EST libraries to ensure the specificity of the target. Two oligonucleotides were synthesized:

M547-569 construct, sense, 5'-TGACAGATAGCCTGAAGC AGTTCAAGAGACTGCTTCAGGCTATCTGTCTTTTTTC-3', antisense, 5'-TCGAGAAAAAAGACAGATAGCCTGAAGCAGT CTCTTGAACTGCTTCAGGCTATCTGTCA-3';

M1294-1316 construct, sense, 5'-TGCGCATCTTCTACTTCA ACTTCAAGAGAGTTGAAGTAGAAGATGCGCTTTTTTC-3', antisense, 5'-TCGAGAAAAAAGCGCATCTTCTACTTCAACTC TCTTGAAGTTGAAGTAGAAGATGCGCA-3'.

C2C12 myoblasts were transfected using Fugene reagent (Roche). Total protein was collected from the RNAi-transfected and empty control cells to identify the silencing of TRPV1 by immunoblotting. RNAi-transfected and control $\mathrm{C} 2 \mathrm{C} 12$ cells were cultured and differentiated to 2-day myotubes for the in vitro study.

\section{Immunoblot analysis}

Cells were treated with capsaicin $(100 \mathrm{nM})$ in the presence or absence of iRTX $(1 \mu \mathrm{M})$ or BAPTA $(10 \mu \mathrm{M})$ for $24 \mathrm{~h}$ before total protein was extracted. Tissues were homogenized and cells were lysed in high-salt extraction buffer $(0.5 \mathrm{~mol} / 1$ Tris, $1 \%$ NP- $40,1 \%$ Triton X-100, $1 \mathrm{~g} / 1$ sodium dodecyl sulfate, $1.5 \mathrm{~mol} / 1 \mathrm{NaCl}, 0.2$ $\mathrm{mol} / 1 \mathrm{EDTA}, 0.01 \mathrm{~mol} / 1 \mathrm{EGTA}$ ) and $0.2 \mathrm{mmol} / 1$ protease inhibitor, placed at $-20{ }^{\circ} \mathrm{C}$ for $20 \mathrm{~min}$ and centrifuged at $12000 \times \mathrm{g}$ at $4{ }^{\circ} \mathrm{C}$ for $20 \mathrm{~min}$ to remove insoluble material. Protein concentration was determined using a DC protein assay kit (Bio-Rad, Hercules, CA, USA). A total of 50- $\mu$ g portions of the protein were resolved on SDS-polyacrylamide gels and electroblotted onto polyvinylidene difluoride membranes. After transfer, the membranes were blocked for $4 \mathrm{~h}$ at room temperature in blocking buffer (Bio-Rad). Next, the membranes were incubated overnight at $4{ }^{\circ} \mathrm{C}$ with antibodies for TRPV1 (Alamone, Israel), PGC-1 $\alpha$ (Cell signaling, USA), CD36, carnitine palmityl transferase 1, hexokinase type II enzyme, surfeit 1 , cytochrome $\mathrm{C} 1$ and $\beta$-actin (Santa Cruz, USA). After 
incubation with secondary antibodies (ZSGB-BIO, China) at room temperature for $2 \mathrm{~h}$, the proteins were detected with enhanced chemiluminescence and quantified using a Gel Doc 2000 Imager (Bio-Rad). Protein expression was normalized to the internal control $\beta$-actin.

\section{Immunofluorescence}

C2C12 myotubes or cryosectioned muscle tissues were fixed with $10 \%$ formalin at room temperature for $60 \mathrm{~min}$, and then bathed in $2 \%$ hydrogen peroxide methanol solution for $30 \mathrm{~min}$. The cells or tissues were washed with water, then blocked with $5 \%$ bovine serum albumin for $20 \mathrm{~min}$ and incubated with TRPV1-specific antibodies (Alamone) overnight at $4{ }^{\circ} \mathrm{C}$. The cells or tissues were then washed three times and incubated with fluorescent dyelabeled secondary antibodies (ZSGB-BIO) at room temperature for $30 \mathrm{~min}$. Control experiments were performed in the absence of primary antibodies. Images were obtained with a TE2000-U Nikon eclipse microscope and analyzed with NIS-Elements imaging software (Nikon, Japan).

\section{Intracellular free calcium imaging}

$\left[\mathrm{Ca}^{2+}\right]_{\mathrm{i}}$ was measured with Fura-2, as described in a previous publication [11]. C2C12 myotubes grown on glass cover slips were loaded with Fura-2 $(2 \mu \mathrm{mol} / \mathrm{l})$ and $0.025 \%$ Pluronic F-127 in physiological saline solution containing $135 \mathrm{mM} \mathrm{NaCl}, 5 \mathrm{mM} \mathrm{KCl}, 1.5$ $\mathrm{mM} \mathrm{CaCl} 2,1 \mathrm{mM} \mathrm{MgCl} 2,11 \mathrm{mM}$ D-glucose and $10 \mathrm{mM}$ HEPES, $\mathrm{pH} 7.4$, for $40 \mathrm{~min}$ at room temperature in the dark. The myotubes were then washed three times with physiological saline solution. Individual cells were defined as the region of interest, and fluorescence was measured using the PTI Fluorescence Master Systems (Photon Technology International, Birmingham, NJ, USA). Fluorescence was measured at $510 \mathrm{~nm}$ emission, with excitation wavelengths of $340 \mathrm{~nm}$ and $380 \mathrm{~nm}$, at baseline and after stimulation with capsaicin with or without pretreatment with iRTX $(1 \mu \mathrm{M})$ for $5 \mathrm{~min}$. Changes in $\left[\mathrm{Ca}^{2+}\right]_{\mathrm{i}}$ were deduced from the ratios of transient increases in fluorescence intensity at $340 \mathrm{~nm}$ and $380 \mathrm{~nm}$.

\section{Mitochondrial staining}

Mitochondria in cultured myotubes were stained with a mitochondrial probe, MitoFluor Red 589, which was reported to accumulate in mitochondria regardless of mitochondrial membrane potential, according to the manufacturer's protocol and previously reported methods [46]. Briefly, C2C12 myotubes were incubated in the prewarmed $\left(37^{\circ} \mathrm{C}\right)$ growth medium containing $200 \mathrm{nM}$ MitoFluor Red 589 for $30 \mathrm{~min}$, and then washed with prewarmed PBS. Images were obtained with a TE2000-U Nikon eclipse microscope (Nikon).

\section{Assessment of ATP production}

ATP contents in $\mathrm{C} 2 \mathrm{C} 12$ myotubes were measured using the ATP Bioluminescence Assay kit (Genmed Scientifics Inc., Shanghai, China) according to the manufacturer's protocol. The cells were kept on ice until assayed for luminescence intensity with Varioskan Flash Type 3001 (Thermo electron Co., Waltham, MA, USA). The values of luminescence intensity were normalized to the protein concentrations.

\section{Generation of TRPV1 transgenic mice}

The transgenic plasmid pcDNA3.1-rTRPV1 was generously provided by Ramon Latorre (Laboratory of Biophysics and Molecular Physiology, Chile). It was generated by cloning a cDNA fragment that encodes the open reading frame of rat TRPV1 into KpnNot sites of pcDNA3.1. The 8.3-kb Pvu fragment of pcDNA3.1rTRPV1 was then isolated and injected into the pronucleus of fertilized oocytes from $\mathrm{C} 57 \mathrm{BL} / 6 \mathrm{~J} \times \mathrm{CBA}$ F1 mice, and the oocytes were implanted into pseudopregnant females. A total of 400 pronuclear injections were performed, resulting in the generation of 120 pups. Tail DNA PCR screening was performed to identify founder mice that harbored the integrated transgene. A screening of the 120 pups identified five pups as founders. This job was completed by the Shanghai Research Center for Biomodel Organism. To maintain an isogenic strain, heterozygous transgenic mice were propagated through breeding with wild-type C57BL/6J mice for two generations. Transgenic mice were identified among the offspring using tail DNA PCR screening. These transgenic mice and their non-transgenic littermates were all fed with a standard laboratory chow. Exercise endurance and oxygen consumption were measured when the mice were 6-month old.

To screen transgenic mice, genomic DNA was purified from tails of 2-week-old mice and used for PCR with the following primers: 5'-GCGTGGATAGCGGTTTGA-3'; 5'CGACTCCTGGATGTGAAGATG-3'. The tail DNA of TRPV1 transgenic mice produced a PCR product of $538 \mathrm{bp}$. TRPV1 protein expression was determined by western blot analysis when the mice were sacrificed at 6-month old.

\section{Animal care}

The C57BL/6J wild-type mice and TRPV1 knockout mice were purchased from Jackson Laboratory (Bar Harbor, Maine, USA). Both types of male mice aged 6-8 weeks were divided into two groups ( $n=12,4$ mice/cage). All mice were housed in colony cages with a 12-h light/12-h dark cycle and allowed unlimited access to water and standard laboratory chow. Control animals were fed with this regular diet alone. Treated animals were fed with this diet supplemented with $0.01 \%$ capsaicin. Body weight was recorded every 10 days. The mean daily food intake was determined the last week of each month by subtracting the amount of food left uneaten each day from the amount provided the previous day. Food spillage into the cage was assessed and found to be negligible. Six mice from each group were kept on their respective diets for 4 months, and then sacrificed for blood and tissue examinations. The remaining six mice in each group were kept on their respective diets for 12 months for exercise endurance tests. The male C57BL/6J mice aged 6-8 weeks were also randomly divided into three groups, respectively, fed with regular chow diet, HFD and HFD plus $0.01 \%$ capsaicin for 4 months. Body weight, running endurance, glucose and insulin tolerance tests and blood parameters were measured. All the animals were cared for and studied according to institutional guidelines after approval from our Institutional Animal Care and Research Advisory Committee.

\section{Exercise endurance tests}

The mice engaged in strenuous exercise on a rodent treadmill (Columbus Instruments, OH, USA) and standard running tests to evaluate maximal running endurance. Prior to the exercise performance test, the mice were acclimated to the treadmill with a 5-min run at $7 \mathrm{~m} / \mathrm{min}$ and a 5 -min run at $10 \mathrm{~m} / \mathrm{min}$ once daily for 2 days. The endurance test regimen was $10 \mathrm{~m} / \mathrm{min}$ for the first $60 \mathrm{~min}$, 
followed by $1 \mathrm{~m} / \mathrm{min}$ increment increases at 15 -min intervals according to the method described in a previous publication [3]. The mice were considered exhausted when they were unable to avoid repeated electrical shocks. Exercise endurance capacity was equated with the total running distance achieved before exhaustion.

\section{Oxygen consumption measurement}

Mice were subjected to oxygen consumption measurements immediately after running for $30 \mathrm{~min}$ at $10 \mathrm{~m} / \mathrm{min}$. Oxygen consumption was measured with a respiration package (Qubit Systems Inc., Canada) consisting of an air pump, a manometer, a metabolic chamber, an oxygen analyzer and a data collecting system. A mouse was placed in the metabolic chamber, and room air was pumped through the chamber. Expired air went through a column filled with a drying agent before it entered the oxygen analyzer. The oxygen percentage data were collected at one point per second and analyzed with the Logger Pro 3.2 Software (Vernier LabPro., Oregon). Oxygen consumption $(\mathrm{ml} / \mathrm{min}$ ) per mouse was calculated as the starting oxygen percentage minus the oxygen percentage at platform multiplied by the air-flow rate $(\mathrm{ml} / \mathrm{min})$. The data are expressed as oxygen consumption per kilogram body weight $(\mathrm{ml} / \mathrm{kg} /$ $\min )$.

\section{Blood analysis}

After fasting for $12 \mathrm{~h}$ and then running for $30 \mathrm{~min}$ at a speed of $10 \mathrm{~m} / \mathrm{min}$, the mice were immediately anesthetized, and blood was collected from the carotid arteries. Serum concentrations of glucose, triglycerides and lactic acid were determined within $24 \mathrm{~h}$ using commercially available assay kits (Applygen Technologies Inc., China). For the mice on HFD, the blood levels of insulin and triglycerides were determined after fasting for $12 \mathrm{~h}$.

\section{Intraperitoneal glucose tolerance test and intraperitoneal insulin tolerance test}

Intraperitoneal glucose tolerance test was carried out in mice on HFD with or without capsaicin supplementation and mice on normal chow diet as previously described [47]. After an overnight fast (14 h), glucose (2 $\mathrm{g} / \mathrm{kg}$ body weight) was administered via injection into the peritoneal cavity, and blood was drawn from the tail vein at $0,30,60$, and $120 \mathrm{~min}$ after glucose administration. Blood glucose levels were determined using the OneTouch Ultra blood glucose meter (LifeScan, CA, USA). Intraperitoneal insulin tolerance test was evaluated in fed mice on a different day. Humulin $\mathrm{R}(0.75 \mathrm{U} / \mathrm{kg}$ body weight) (Eli Lilly and Co., IN, USA) in sterile saline was administered intraperitoneally. Glucose levels were determined in the tail blood at 0, 15, 30 and $60 \mathrm{~min}$ after insulin injection.

\section{Muscle fiber typing}

The gastrocnemius muscle was immediately extracted and stored in liquid nitrogen. Muscle fiber typing was performed using metachromatic dye-ATPase methods, as described in a previous publication [48].

\section{Muscular lipid contents determination}

Lipid accumulation in muscles was assessed by Oil-Red O staining [49]. Briefly, a stock solution was prepared by solubilizing $300 \mathrm{mg}$ of oil red O dye (Sigma, USA) in $100 \mathrm{ml}$ of concentrated isopropanol (99\%). Cryosectioned muscle tissues were incubated at room temperature for $10 \mathrm{~min}$ in $12 \mathrm{ml}$ of freshly prepared stock solution (filtered through a Whatmann paper) diluted in $8 \mathrm{ml}$ of distilled water, and then washed with running water for $10 \mathrm{~min}$. Images were obtained with a TE2000-U Nikon eclipse microscope and lipid droplet area was analyzed with NIS-Elements imaging software (Nikon).

\section{Electron microscopic observation of mitochondria}

Fresh skeletal muscles were cut into $1 \mathrm{~mm}$ cubes, which were fixed in $3 \%$ glutaraldehyde for $2 \mathrm{~h}$, and then fixed in $1 \%$ osmium tetroxide, stepwise dehydrated in graded acetone, and infiltrated, embedded and polymerized in EPON 812. The semi-thin sections were optically positioned and further sectioned with ultramicrotome into 50-60 $\mathrm{nm}$ pieces, which were collected on copper grids, double-stained with uranyl acetate and lead citrate and then observed under Hitachi H-600IV transmission electron microscope and photographed. Mitochondrial areas were calculated with NISElements imaging software (Nikon).

\section{Mitochondrial respiration measurement}

Mitochondria were isolated from fresh quadriceps femoris muscles by using a mitochondria isolation kit (Genmed Scientifics Inc.). The respiratory activity of the mitochondria was potentiometrically measured at $27 \mathrm{C}$, in an oxygraph vessel with a Clark electrode (Yellow Springs Instruments, OH, USA). Mitochondria were incubated in a medium containing $75 \mathrm{mmol} / \mathrm{l}$ mannitol, 25 mmol/1 sucrose, $100 \mathrm{mmol} / 1 \mathrm{KCl}, 50 \mu \mathrm{mol} / 1 \mathrm{EDTA}, 5 \mathrm{mmol} /$ $1 \mathrm{~K}_{2} \mathrm{HPO}_{4}$ and $5 \mathrm{mmol} / 1$ Tris- $\mathrm{HCl}, \mathrm{pH}$ 7.35. The substrates used were $6 \mathrm{mmol} / 1$ sodium pyruvate and $6 \mathrm{mmol} / 1$ sodium malate. State 3 oxygen consumption was measured in the presence of 0.6 $\mathrm{mmol} / \mathrm{l} \mathrm{ADP}$ and state 4 oxygen consumption in the absence of ADP. The values were normalized to mitochondrial protein contents. Respiratory control ratio was calculated from the value of state 4 relative to state 3 .

\section{Statistics}

Data are expressed as the mean \pm S.E.M. Statistical differences between groups were assessed by Student's $t$-test or one-way analysis of variance with Bonferroni's multiple comparison post hoc tests, as appropriate. Two-sided $P$-values below 0.05 were considered statistically significant.

\section{Acknowledgments}

We acknowledge Dr R Latorre (Laboratory of Biophysics and Molecular Physiology, Chile) for the gift of plasmid pcDNA3.1rTRPV1. We thank Lijuan Wang (Chongqing Institute of Hypertension, China) for technical assistance and Wing Tak Wong (Chinese University of Hong Kong, China) for revision of the manuscript. This research was supported by grants from the 973 Program (2012CB517805 and 2011CB503902) and the National Natural Science Foundation (31000519 and 30890042).

\section{References}

1 Yung LM, Laher I, Yao X, Chen ZY, Huang Y, Leung FP. Exercise, vascular wall and cardiovascular diseases: an update (part 2). Sports Med 2009; 39:45-63.

2 Smith AG, Muscat GE. Skeletal muscle and nuclear hormone 
receptors: implications for cardiovascular and metabolic disease. Int J Biochem Cell Biol 2005; 37:2047-2063.

3 Wang YX, Zhang CL, Yu RT, et al. Regulation of muscle fiber type and running endurance by PPARdelta. PLoS Biol 2004; 2:e294.

4 Ryder JW, Bassel-Duby R, Olson EN, Zierath JR. Skeletal muscle reprogramming by activation of calcineurin improves insulin action on metabolic pathways. J Biol Chem 2003; 278:44298-44304.

5 Luquet S, Lopez-Soriano J, Holst D, et al. Peroxisome proliferator-activated receptor delta controls muscle development and oxidative capability. FASEB J 2003; 17:2299-2301.

6 Jeukendrup AE, Saris WH, Wagenmakers AJ. Fat metabolism during exercise: a review--part III: effects of nutritional interventions. Int J Sports Med 1998; 19:371-379.

7 Caterina MJ, Schumacher MA, Tominaga M, Rosen TA, Levine JD, Julius D. The capsaicin receptor: a heat-activated ion channel in the pain pathway. Nature 1997; 389:816-824.

8 Pedersen SF, Owsianik G, Nilius B. TRP channels: an overview. Cell Calcium 2005; 38:233-252.

9 Caterina MJ. Vanilloid receptors take a TRP beyond the sensory afferent. Pain 2003; 105:5-9.

10 Xin H, Tanaka H, Yamaguchi M, Takemori S, Nakamura A, Kohama K. Vanilloid receptor expressed in the sarcoplasmic reticulum of rat skeletal muscle. Biochem Biophys Res Commun 2005; 332:756-762.

11 Zhang LL, Yan Liu D, Ma LQ, et al. Activation of transient receptor potential vanilloid type-1 channel prevents adipogenesis and obesity. Circ Res 2007; 100:1063-1070.

12 Yang D, Luo Z, Ma S, et al. Activation of TRPV1 by dietary capsaicin improves endothelium-dependent vasorelaxation and prevents hypertension. Cell Metab 2010; 12:130-141.

13 Kim KM, Kawada T, Ishihara K, Inoue K, Fushiki T. Increase in swimming endurance capacity of mice by capsaicininduced adrenal catecholamine secretion. Biosci Biotechnol Biochem 1997; 61:1718-1723.

14 Oh TW, Ohta F. Dose-dependent effect of capsaicin on endurance capacity in rats. Br J Nutr 2003; 90:515-520.

15 Shin KO, Moritani T. Alterations of autonomic nervous activity and energy metabolism by capsaicin ingestion during aerobic exercise in healthy men. J Nutr Sci Vitaminol (Tokyo) 2007; 53:124-132.

16 Lin J, Wu H, Tarr PT, et al. Transcriptional co-activator PGC1 alpha drives the formation of slow-twitch muscle fibres. Nature 2002; 418:797-801.

17 Naya FJ, Mercer B, Shelton J, Richardson JA, Williams RS, Olson EN. Stimulation of slow skeletal muscle fiber gene expression by calcineurin in vivo. $J$ Biol Chem 2000; 275:45454548.

$18 \mathrm{Wu}$ H, Kanatous SB, Thurmond FA, et al. Regulation of mitochondrial biogenesis in skeletal muscle by CaMK. Science 2002; 296:349-352.

19 Handschin C, Chin S, Li P, et al. Skeletal muscle fibertype switching, exercise intolerance, and myopathy in PGC1alpha muscle-specific knock-out animals. J Biol Chem 2007; 282:30014-30021.

20 Kusuhara K, Madsen K, Jensen L, Hellsten Y, Pilegaard H. Calcium signalling in the regulation of PGC-1alpha, PDK4 and HKII mRNA expression. Biol Chem 2007; 388:481-488.
21 Guerfali I, Manissolle C, Durieux AC, Bonnefoy R, Bartegi A, Freyssenet D. Calcineurin A and CaMKIV transactivate PGC1alpha promoter, but differentially regulate cytochrome C promoter in rat skeletal muscle. Pflugers Arch 2007; 454:297305.

22 Pilegaard H, Saltin B, Neufer PD. Exercise induces transient transcriptional activation of the PGC-1alpha gene in human skeletal muscle. J Physiol 2003; 546:851-858.

23 Bruce CR, Hoy AJ, Turner N, et al. Overexpression of carnitine palmitoyltransferase- 1 in skeletal muscle is sufficient to enhance fatty acid oxidation and improve high-fat dietinduced insulin resistance. Diabetes 2009; 58:550-558.

24 Holloway GP, Luiken JJ, Glatz JF, Spriet LL, Bonen A. Contribution of FAT/CD36 to the regulation of skeletal muscle fatty acid oxidation: an overview. Acta Physiol (Oxf) 2008; 194:293-309.

25 Burgess SC, He T, Yan Z, et al. Cytosolic phosphoenolpyruvate carboxykinase does not solely control the rate of hepatic gluconeogenesis in the intact mouse liver. Cell Metab 2007; 5:313-320.

26 Burgess SC, Hausler N, Merritt M, et al. Impaired tricarboxylic acid cycle activity in mouse livers lacking cytosolic phosphoenolpyruvate carboxykinase. J Biol Chem 2004; 279:48941-48949.

27 Hakimi P, Yang J, Casadesus G, et al. Overexpression of the cytosolic form of phosphoenolpyruvate carboxykinase (GTP) in skeletal muscle repatterns energy metabolism in the mouse. J Biol Chem 2007; 282:32844-32855.

28 Wende AR, Schaeffer PJ, Parker GJ, et al. A role for the transcriptional coactivator PGC-1alpha in muscle refueling. $J$ Biol Chem 2007; 282:36642-36651.

29 Ahn KJ, Hwang HS, Park JH, et al. Evaluation of the role of hexokinase type II in cellular proliferation and apoptosis using human hepatocellular carcinoma cell lines. $\mathrm{J} \mathrm{Nucl} \mathrm{Med}$ 2009; 50:1525-1532.

30 Sparks LM, Xie H, Koza RA, et al. A high-fat diet coordinately downregulates genes required for mitochondrial oxidative phosphorylation in skeletal muscle. Diabetes 2005; 54:19261933.

31 Joyner MJ, Coyle EF. Endurance exercise performance: the physiology of champions. J Physiol 2008; 586:35-44.

32 Erikssen G, Liestol K, Bjornholt J, Thaulow E, Sandvik L, Erikssen J. Changes in physical fitness and changes in mortality. Lancet 1998; 352:759-762.

33 Kokkinos P, Myers J, Kokkinos JP, et al. Exercise capacity and mortality in black and white men. Circulation 2008; 117:614-622.

34 Sandvik L, Erikssen J, Thaulow E, Erikssen G, Mundal R, Rodahl K. Physical fitness as a predictor of mortality among healthy, middle-aged Norwegian men. N Engl J Med 1993; 328:533-537.

35 Kark T, Bagi Z, Lizanecz E, et al. Tissue-specific regulation of microvascular diameter: opposite functional roles of neuronal and smooth muscle located vanilloid receptor-1. Mol Pharmacol 2008; 73:1405-1412.

36 Yoshioka T, Inagaki K, Noguchi T, et al. Identification and characterization of an alternative promoter of the human PGC-1alpha gene. Biochem Biophys Res Commun 2009; 381:537-543. 
37 Jeukendrup AE, Saris WH, Wagenmakers AJ. Fat metabolism during exercise: a review--part II: regulation of metabolism and the effects of training. Int J Sports Med 1998; 19:293302.

38 Tanner CJ, Barakat HA, Dohm GL, et al. Muscle fiber type is associated with obesity and weight loss. Am J Physiol Endocrinol Metab 2002; 282:E1191-1196.

39 Hickey MS, Carey JO, Azevedo JL, et al. Skeletal muscle fiber composition is related to adiposity and in vitro glucose transport rate in humans. Am J Physiol 1995; 268:E453-457.

40 Calvo JA, Daniels TG, Wang X, et al. Muscle-specific expression of PPARgamma coactivator-1alpha improves exercise performance and increases peak oxygen uptake. J Appl Physiol 2008; 104:1304-1312.

41 Wenz T, Rossi SG, Rotundo RL, Spiegelman BM, Moraes CT. Increased muscle PGC-1alpha expression protects from sarcopenia and metabolic disease during aging. Proc Natl Acad Sci USA 2009; 106:20405-20410.

42 Suh YG, Oh U. Activation and activators of TRPV1 and their pharmaceutical implication. Curr Pharm Des 2005; 11:26872698.

43 Kunos G, Osei-Hyiaman D, Liu J, Godlewski G, Batkai S. Endocannabinoids and the control of energy homeostasis. $J$ Biol Chem 2008; 283:33021-33025.
44 Cavuoto P, McAinch AJ, Hatzinikolas G, Janovska A, Game P, Wittert GA. The expression of receptors for endocannabinoids in human and rodent skeletal muscle. Biochem Biophys Res Commun 2007; 364:105-110.

45 Lizanecz E, Bagi Z, Pasztor ET, et al. Phosphorylation-dependent desensitization by anandamide of vanilloid receptor-1 (TRPV1) function in rat skeletal muscle arterioles and in Chinese hamster ovary cells expressing TRPV1. Mol Pharmacol 2006; 69:1015-1023.

46 Diwan A, Matkovich SJ, Yuan QY, et al. Endoplasmic reticulum-mitochondria crosstalk in NIX-mediated murine cell death. J Clin Invest 2009; 119:203-212.

47 Sun C, Zhang F, Ge X, et al. SIRT1 improves insulin sensitivity under insulin-resistant conditions by repressing PTP1B. Cell Metab 2007; 6:307-319.

48 Ogilvie RW, Feeback DL. A metachromatic dye-ATPase method for the simultaneous identification of skeletal muscle fiber types I, IIA, IIB and IIC. Stain Technol 1990; 65:231241.

49 Lee S, Guerra N, Arslanian S. Skeletal muscle lipid content (SMLC) in black and white over-weight youth: an independent risk factor of insulin resistance. Diabetes 2008; 57:A493A494.

(Supplementary information is linked to the online version of the paper on the Cell Research website.) 